De la Fe, C., Vidaurreta, I., Gómez, A. \& Corrales, J.C. (2015). El método de estudio de casos: Una herramienta docente válida para la adquisición de competencias. Revista Electrónica Interuniversitaria de Formación del Profesorado, 18 (3), 127-136.

\title{
El método de estudio de casos: Una herramienta docente válida para la adquisición de competencias
}

\author{
Christian de la Fe Rodríguez ${ }^{(1)}$, Irene Vidaurreta Porrero ${ }^{(2)}$, Ángel Gómez Martín ${ }^{(1)}$, Juan \\ Carlos Corrales Romero ${ }^{(1)}$ \\ ${ }^{(1)}$ Departamento de Sanidad Animal, Facultad de Veterinaria, Universidad de Murcia \\ ${ }^{(2)}$ Cuerpo de Profesores Interinos de Educación Secundaria, Región de Murcia
}

\section{Resumen}

En el contexto actual de la enseñanza, el profesor ha dejado fundamentalmente de transmitir conocimientos para pasar a involucrarse de una forma mucho más activa en el proceso de aprendizaje, cuyo papel fundamental debe recaer en el alumno. La aplicación del método de estudio de casos facilita que éstos asuman el papel de protagonistas activos de su propio aprendizaje, potenciando la adquisición de competencias disciplinares, genéricas y académicas. En este trabajo, presentamos las experiencias realizadas en dos contextos educativos muy diferentes: la docencia práctica de la asignatura de Enfermedades Infecciosas de la materia de Sanidad Animal en la Licenciatura de Veterinaria y en segundo lugar, exponemos su utilización como herramienta en las clases teóricas de la asignatura de Economía de $1^{\circ}$ y $2^{\circ}$ de Bachiller. Este trabajo presenta las pautas generales de la metodología utilizada y las principales conclusiones obtenidas de su aplicación en el proceso de aprendizaje.

\section{Palabras clave}

Aprendizaje basado en problemas, método de casos, Economía, Enfermedades Infecciosas

\section{The case study method: A valuable teaching tool for the acquisition of skills}




\section{Abstract}

In the current context of education, the teacher has essentially stopped transmitting knowledge to get involved more actively in the learning process, whose key role lies with the student. The application of the case study method enables them to assume the role of active protagonists of their own learning, promoting the acquisition of disciplinary, generic and academic competitions. In this paper, we present the experiments carried out in two very different educational contexts: the practical teaching of the subject matter Infectious diseases of Animal Health in the Bachelor of Veterinary and secondly, we present its use as a tool in the theoretical classes the subject of economics 1st and 2nd. This paper presents the general guidelines of the methodology used and the main conclusions of its application in the learning process.

\section{Key words}

Problem based learning, case studies, Economy, Infectious diseases

\section{Introducción}

El modelo educativo que defiende el Espacio Europeo de Educación Superior (EEES), centrado en el aprendizaje de competencias ha implicado necesariamente el cambio de papel del profesor. En este sentido, el docente ha dejado fundamentalmente de transmitir conocimientos para pasar a involucrarse de una forma mucho más activa en el proceso de aprendizaje. Entre las competencias actuales del docente hay que citar el organizar y moderar situaciones de aprendizaje, gestionar la progresión de los mismos, concebir y promover la evolución de dispositivos de diferenciación, implicar a los alumnos en sus aprendizajes y su trabajo, fomentar el trabajo en equipo o utilizar nuevas tecnologías (Perrenoud, 2004).

En este contexto, la utilidad del empleo de metodologías de aprendizaje activas como el aprendizaje basado en problemas (APP) ha resultado exitoso en función de los resultados obtenidos en diferentes experiencias en diferentes ámbitos educativos (Fernández et al., 2006). En el APP, el punto de partida es un problema o situación que permite al estudiante identificar necesidades para comprender mejor ese problema/situación, identificar principios que sustentan el conocimiento y cumplir objetivos de aprendizaje relacionados a cada porción del programa educacional (Branda, 2004). Esta metodología se realiza mayoritariamente a través del trabajo en grupos tutorizados y del trabajo individual autodirigido, con la finalidad de combinar la adquisición de conocimientos con el desarrollo de habilidades generales y actitudes útiles para el ámbito profesional (Fernández et al., 2006). Así, es interesante mencionar que diversos autores indican que su empleo mejora ostensiblemente la adquisión de las competencias transversales (Jiménez et al., 2013).

Inicialmente, se ha empleado en Ciencias de la Salud, iniciando el procedimiento la Facultad de Medicina de la Universidad de Cleveland (USA), en 1950. Con posterioridad, la Universidad de Mc Máster (Canadá) inició lo que hoy conocemos como aprendizaje por problemas (APP). En este método, las ciencias básicas y las clínicas forman un todo, dado que las competencias exigidas al futuro profesional se fundamentan en ambas (AlfonsoRoca y Fonseca, 2001). No obstante, son muchas las carreras universitarias fuera del ámbito clínico donde ya se emplea esta metodología (Mateo y Vlachopoulos, 2012)

No obstante, la aplicación de este tipo de metodologías educativas en la esfera educativa universitaria también plantea interrogantes de otro tipo, como podría ser la ruptura con la metodología aplicada en los niveles educativos previos como el bachillerato o la educación 
secundaria. Por ello, consideramos que siempre es interesante exponer las experiencias desarrolladas en ámbitos educativos muy diferentes, al objeto de poder realizar una comparativa en la evolución del alumnado que ha trabajado anteriormente siguiendo un APP y que continua sirviéndose de él en su etapa universitaria y los que se enfrentan a ella por primera vez. Evidentemente, es del todo inapropiado tratar de comparar explícitamente los resultados del aprendizaje, pero si es interesante evaluar la existencia de problemas comunes que permitan la adopción de medidas correctoras apropiadas a cada caso.

En este trabajo, exponemos dos experiencias de aplicación de dicho método en dos contextos educativos muy diferentes entre sí:

Por un lado, exponemos su utilización para la docencia práctica de la asignatura de Enfermedades Infecciosas de la materia de Sanidad Animal en la Licenciatura de Veterinaria. En esta asignatura clínica de cuarto curso, la aplicación disciplinar del citado método ha permitido ir incorporando progresivamente un sistema de aprendizaje autónomo y significativo a las prácticas ya incorporado con posterioridad a la docencia del Grado de Veterinaria, que responde técnicamente al citado método de estudio de casos. En este sentido, es necesario aclarar que en la literatura de la pedagogía médica existen al menos tres grandes grupos de métodos que utilizan el abordaje de problemas clínicos: 1) Casos clínicos asociados a una clase teórica, 2) aprendizaje por problemas, caracterizado por su filosofía y abordaje multidisciplinar y 3) el método de estudio de caso, caracterizado por la presentación de un caso clínico, centrado en la disciplina objeto de estudio. Utilizando la filosofía del APP, pero sin la exigencia de realizar un abordaje multidisciplinar, va a ser este último, el estudio de caso, el tipo el que más facilidades entraña para su aplicación inmediata en cualquier disciplina. El planteamiento necesita forzosamente que el alumno realice la aplicación simultánea de conocimientos previamente aprendidos y de aquellos mostrados por primera vez (Wassermann, 1994), y exige que éstos asuman el papel de protagonistas activos en vez de mantener una actitud pasiva y, por tanto, salven la distancia entre teoría y práctica. Adicionalmente, crea un diálogo en el grupo que rompe con los esquemas tradicionales de la clase teórica, implica al alumnado en su propio aprendizaje y posibilita la discusión y el trabajo en equipo.

En segundo lugar, exponemos su utilización como herramienta en las clases teóricoprácticas de la asignatura de Economía de $1^{\circ}$ y $2^{\circ}$ de Bachiller, que si bien se trata de una asignatura de Ciencias Sociales, se presta muy adecuadamente a la puesta en práctica de del sistema APP. La aplicación de este tipo de métodos fuera del contexto clínico originario constituye un verdadero reto para el docente, y aquí se expone la experiencia y su efecto en la docencia impartida. Dos factores son importantes a considerar para valorar la experiencia realizada: Su empleo en Educación Secundaria y en una materia evidentemente no clínica. Por las características de la asignatura de Economía de los diferentes cursos de Bachillerato en los que se imparte, la aplicación del método de casos en cada uno de ellos presenta matices diferenciadores que exponemos a continuación en el material y métodos del trabajo.

El listado de participantes en ambas experiencias se muestras en la Tabla 1. 
Tabla 1.

Distribución de alumnos participantes por experiencia

\begin{tabular}{|l|l|l|l|}
\hline Titulación & $\begin{array}{l}\text { Número } \\
\text { total }\end{array}$ & $\begin{array}{l}\text { Módulos/ } \\
\text { U.D.** }\end{array}$ & $\begin{array}{l}\text { Grupos de } \\
\text { trabajo/problema } \\
*\end{array}$ \\
\hline $\begin{array}{l}\text { Enfermedades Infecciosas (4 curso de la } \\
\text { Licenciatura de Veterinaria) }\end{array}$ & 100 & 20 & $3-4$ \\
\hline Economía (1 ${ }^{\circ}$ curso de Bachiller) & 18 & 18 & $4-3$ \\
\hline Economía (2 ${ }^{\circ}$ curso de Bachiller) & 17 & 12 & $4-7$ \\
\hline
\end{tabular}

*Caso clínico en la experiencia realizada en la Licenciatura de Veterinaria

**Unidades didácticas en Bachillerato

\section{Metodología}

En la primera experiencia, desarrollada en la asignatura de Enfermedades Infecciosas de la Licenciatura de Veterinaria, el profesor divide los alumnos en grupos de 3-4 personas y a continuación aporta los casos a estudiar (del ámbito de las enfermedades infecciosas) la guía para el análisis y diferentes fuentes de información (libros, revistas especializadas, guías docentes e Internet), que los alumnos consultarán según su propio criterio, para analizar el caso y establecer las hipótesis de trabajo. Es importante mencionar que el alumno también dispone del resto de las herramientas necesarias para el diagnóstico (medios de cultivo, pruebas bioquímicas, etc.), las cuales sólo le serán aportadas por el profesor cuando se requieran. De este modo, los alumnos, realizan una labor autónoma para la resolución de los casos, tomando las decisiones que consideran más oportunas para la resolución de los mismos tras la revisión crítica de la anamnesis y de las muestras remitidas. Tras realizar el diagnóstico, deben prescribir las medidas terapéuticas y de control de cada caso.

Al objeto de recabar información en referencia a la adquisición de competencias, realizamos la puesta en común de cada caso donde el profesor actúa como coordinador y dirige la puesta en común de los casos por parte de los grupos de trabajo, fomentando la participación y la discusión y aportando su opinión sobre la resolución del caso. Es importante indicar que aunque los alumnos estén divididos en grupos, el desarrollo del trabajo aporta al profesor los argumentos suficientes para valorar individualmente la adquisición de competencias genéricas como el liderazgo o la toma de decisiones. De modo adicional, los estudiantes complementaron un cuestionario de autoevaluación y evaluación recíproca orientado a valorar su labor y la de todos los componentes del grupo. Los criterios a valorar fueron:
1. Asistencia y puntualidad
2. Conocimientos teóricos previos
3. Aportación de ideas
4. Aprovechamiento del tiempo
5. Gestión de la información 


\section{Resolución de problemas \\ 7. Capacidad organizativa \\ 8. Coherencia en la evaluación grupal}

En la segunda experiencia, llevada a cabo en la asignatura de Economía de $1^{\circ}$ de Bachillerato, el docente planteó a lo largo del curso 3 situaciones problemáticas para su resolución mediante el trabajo en equipos formados por equipos de entre tres a cuatro componentes; a) en primer lugar, el objetivo consistía en profundizar en el conocimiento de las instituciones internacionales y de ciertos países extranjeros que por su situación económica pudieran resultar de relevancia para la economía española, para ello se presentó, en primer lugar, un cuestionario al que los alumnos debían responder sirviéndose tanto de internet como de los medios informativos a fin de determinar cuáles eran esas instituciones y países y en segundo lugar, se pretendía que formulasen en grupo una demanda por grupo de países ante las Naciones Unidas, a fin de que investigasen sobre las necesidades, intereses, instituciones, dirigentes y relaciones internacionales de los países objeto de estudio b) el segundo caso, llevado a cabo conjuntamente con la asignatura de lengua española, pretendía generar conocimiento sobre la situación socio-económica más próxima, estableciendo una relación con las decisiones políticas nacionales y su entorno más inmediato, mediante la creación de un periódico impreso cuya financiación corría a cargo de anuncios publicitarios que ellos mismos se encargaron de buscar, y que se llevó a cabo realmente y no solo en el plano teórico, para ello los alumnos por grupos de entre 3 y cuatro miembros eligieron un tema de interés y realizaron una serie de entrevistas a empresarios, políticos e investigadores de su entorno, c) y el tercer y último caso consistía en la realización de un análisis por grupos de 3 a 4 miembros, de las estrategias de mercado de una empresa elegida por ellos, que luego debieron exponer mediante PowerPoint en el mismo aula.

Estas tres experiencias fueron evaluadas de forma grupal atendiendo a los objetivos y contenidos expuestos y posteriormente mediante test personales sobre los conocimientos adquiridos en el desarrollo de cada experiencia y la implicación personal de cada alumno.

En la asignatura de Economía de la Empresa de $2^{\circ}$ de Bachillerato, el empleo del método APP quedó más limitado a la aplicación práctica directa del contenido de la asignatura, llevando a cabo simulaciones repetidas de las pruebas de selectividad a las que habrían de hacer frente los alumnos al concluir el curso académico. Así pues, se planteó el trabajo en equipo en grupos de 3 a 4 miembros para afrontar la resolución de los casos prácticos. En este contexto formativo, se ofreció a los alumnos una batería de casos prácticos para su resolución por equipos, que posteriormente eran puestos en común, en esta dinámica el docente toma el rol de coordinador-moderador para así favorecer la participación de todos, aportando finalmente su opinión del caso. En la evaluación periódica llevada a cabo a lo largo del cuso se evaluaba el efecto de esta experiencia mediante test personales y el planteamiento de casos para su resolución individual.

\section{Discusión y conclusiones}

Los resultados de aprendizaje de ambas experiencias deben considerarse como muy positivas, basándonos en la evaluación de las actividades desarrolladas.

En la experiencia realizada en el ámbito universitario, la aplicación del método de estudio de casos en las prácticas de diagnóstico de Enfermedades Infecciosas ha facilitado que los 
alumnos asuman el papel de protagonistas activos de su propio aprendizaje. El empleo de la misma en el contexto de una asignatura concreta, cuyo posible uso ya fue planteado con anterioridad (De la Fe y Corrales, 2007) permite tanto que las competencias profesionales respondan principalmente a los definidos por la disciplina como que las competencias profesionales objeto de estudio y los problemas como herramientas del mismo no presenten necesariamente un carácter multidisciplinar. Ello podría parecer un problema si analizamos exclusivamente el concepto de APP, pero en nuestro caso, consideramos que el método de casos permite ir adaptando progresivamente a docencia hacia el necesario carácter multidisciplinar que finalmente deben caracterizarla.

Entre los aspectos más positivos de la experiencia confirmamos el papel activo del estudiante en el proceso de aprendizaje, ya que tras una fase inicial de cierto desconcierto, los estudiantes participantes en la experiencia son capaces de establecer una hipótesis inicial de trabajo y confirmarla con posterioridad a lo largo de los días de prácticas mediante el empleo de una metodología adecuada a los objetivos concretos de cada caso. Más allá de los propios resultados de los casos asignados, el estudiante adquiere la metodología general de trabajo, mediante el desarrollo combinado de trabajo autónomo y grupal, como lo demuestran las exposiciones orales y el trabajo diario realizado durante los días de la experiencia. Otro aspecto muy destacable es el establecimiento de foros de discusión entre los propios grupos o en el seno de ellos individualmente. Independientemente de la calificación obtenida en las prácticas, cuya media oscila entre 7,5 sobre 10 puntos, los estudiantes perciben positivamente el empleo de este tipo de metodologías activas en el desarrollo de sus prácticas, tal y como pudimos comprobar tras el desarrollo de cada sesión. Todos estos aspectos, coinciden en líneas generales con lo observado por otros autores (Fernández et al., 2006).

Otro aspecto interesante de la experiencia realizada es la valoración del empleo de esta metodología para la adquisición de las competencias transversales como la capacidad de liderazgo, la organización, el empleo de la lengua inglesa o, evidentemente, la resolución de problemas. En este sentido, su empleo es muy válido para su aprendizaje y su valoración. Para ello, podemos valorar también el empleo del cuestionario de autoevaluación y la evaluación recíproca. Un ejemplo de los resultados obtenidos podemos observarlo en la Figura 1, donde se indican las calificaciones otorgadas por los estudiantes a sí mismo (Alumno 1) y al resto de componentes de su grupo (Alumnos 2 a 4) para un total de 8 items con 8 puntos a repartir para todo el grupo en cada uno de ellos.

En líneas generales, podemos distribuir a los alumnos en tres grupos en función de los resultados de la autoevaluación y la evaluación recíproca. La evaluación máxima era 8 puntos y los grupos los siguientes:

a) Alumnos que se califican mejor (5 o más puntos) al resto del grupo: $31.5 \%$

b) Alumnos que califican mejor a otro compañero del grupo (5 o más puntos): 10.5\%

c) Alumnos que califican igual a todos los miembros ( 5 o menos puntos): $58 \%$ 


\begin{tabular}{c|c|c|c|c|c} 
Criterios & Alumno 1 & Alumno 2 & Alumno 3 & Alumno 4 & $\begin{array}{c}\text { Puntos a } \\
\text { repartir }\end{array}$ \\
\hline C1 & 2 & 2 & 2,3 & 1,7 & 8 \\
\hline C2 & 2,1 & 2 & 2,3 & 1,6 & 8 \\
\hline C3 & 2,4 & 1,8 & 2,5 & 1,3 & 8 \\
\hline C4 & 2,3 & 1,6 & 2,4 & 1,7 & 8 \\
\hline C5 & 2,4 & 2,1 & 2,5 & 1 & 8 \\
\hline C6 & 2 & 2 & 2 & 2 & 8 \\
\hline C7 & 2,4 & 1,9 & 2,4 & 1,1 & 8 \\
\hline C8 & 2,1 & 2,3 & 2,3 & 1,3 & 8 \\
\hline
\end{tabular}

Figura 1. Ejemplo de cuestionario de autoevaluación y evaluación recíproca

En este sentido, observamos que los alumnos son reticentes a otorgar una calificación superior en términos globales a otro miembro de su grupo, y en la mayor parte de los casos, la evaluación recíproca entre los miembros del grupo es similar, lo cual no tiene repercusión en la calificación. No obstante, detectamos la presencia de un grupo de encuestados que valora mejor a otros compañeros, lo cual, desde el punto de vista objetivo, permite valorar positivamente la primera aplicación de esta herramienta docente en las prácticas de Enfermedades Infecciosas I. Además, si analizamos la valoración individual en algunos de estos ítems (como la aportación de ideas o la resolución de problemas) otorgado tanto por el profesor como por los estudiantes de cada grupo, las calificaciones superiores generalmente se otorgan a los mismos estudiantes, en coincidencia con lo observado por otros autores (Jiménez et al., 2013)

El uso del $A B P$ en Economía uso se ha realizado en ocasiones en materias relacionadas en el contexto universitario (Jiménez et al., 2013), si bien su uso fuera del contexto universitario supone siempre un verdadero reto. Así, en $1^{\circ}$ de Bachillerato, los resultados obtenidos permitieron comprobar el establecimiento de un incremento en el conocimiento de la situación económica general que de otro modo no se hubiese asimilado tan vivamente. Los resultados obtenidos pueden observarse en la siguiente tabla:

Tabla 2.

Calificaciones obtenidas en $1^{\circ}$ de Bachillerato

\begin{tabular}{|c|c|}
\hline & $\begin{array}{l}\text { CALIFICACIÓN } \\
\text { MEDIA }\end{array}$ \\
\hline CASO 1 (1. EVALUACION) & 6.67 \\
\hline $1^{\mathrm{a}}$ EVALUACION & 5.84 \\
\hline CASO 2 ( $2^{\mathrm{a}}$ EVALUACION $)$ & 8.35 \\
\hline $2^{\mathrm{a}}$ EVALUACION & 5.74 \\
\hline CASO 3 ( $3^{3}$ EVALUACION $)$ & 6.79 \\
\hline $3^{\mathrm{a}}$ EVALUACION & 6.85 \\
\hline
\end{tabular}


En esta experiencia pudieron observarse 2 conclusiones:

1. El método de APP permite reforzar los conocimientos de la materia.

2. El método de APP influye directamente en la motivación de los alumnos.

3. Una actividad planteada de acuerdo con el método de APP, puede llegar a interferir negativamente en el tiempo dedicado al estudio tradicional para preparar un examen según una metodología convencional, si llega a ser suficientemente emocionante para los alumnos como ocurrió en el

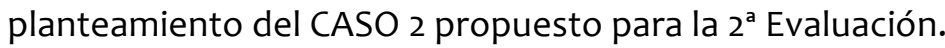

En $2^{\circ}$ de Bachillerato, tal y como ya se indicó, al aprendizaje orientado a una prueba de Selectividad permitió deducir que los resultados dependían no solo de los métodos utilizados sino de la implicación personal de los alumnos. Para Economía de la Empresa se plantearon 6 casos para trabajar en grupo, para cuya resolución contaban con el material aportado por el profesor. La valoración de los alumnos en estos planteamientos se recoge en la siguiente tabla:

Tabla 3.

\begin{tabular}{|c|c|}
\hline & $\begin{array}{l}\text { CALIFICACIÓN } \\
\text { MEDIA (S/10) }\end{array}$ \\
\hline CASO $1\left(1^{a}\right.$ EVALUACION $)$ & 7.35 \\
\hline $1^{\mathrm{a}}$ EVALUACION & 6.23 \\
\hline CASO $2\left(2^{\mathrm{a}}\right.$ EVALUACION $)$ & 7.47 \\
\hline CASO $3\left(2^{\mathrm{a}}\right.$ EVALUACION) & 7.08 \\
\hline $2^{\mathrm{a}}$ EVALUACION & 5.63 \\
\hline CASO 4 ( $3^{\mathrm{a}}$ EVALUACION) & 7.85 \\
\hline CASO 5 ( $3^{\mathrm{a}}$ EVALUACION $)$ & 6.89 \\
\hline CASO 6 ( $3^{\mathrm{a}}$ EVALUACION) & 6.95 \\
\hline $3^{\text {a EVALUACION }}$ & 5.91 \\
\hline
\end{tabular}

En la presente tabla se observa una valoración en la que se constatan los siguientes hechos:

1. Es el reflejo evidente del incremento de la complejidad en los casos planteados.

2. El trabajo en equipo favorecido por el método de APP favorece la resolución positiva de los casos.

3. El método de APP puede llegar a excluir a algunos miembros del equipo de su participación en la resolución del caso, ya sea por decisión propia o por la de los restantes miembros, lo que ocasiona el desinterés de esos miembros por la asignatura.

4. Al tratarse la asignatura de Economía de la Empresa de una materia optativa en las pruebas de selectividad, se aprecia una relajación en las calificaciones finales de los que al final de la $3^{\text {a }}$ Evaluación ya han decidido no presentarse al examen de selectividad con esta asignatura. 
No obstante, en ambas experiencias los casos se proponen a los grupos de alumnos para que individual o colectivamente se enfrenten al problema y tomen decisiones. El caso se utiliza, pues, para implicar al estudiante, que adopta el rol de protagonista de la acción: analiza la situación, define el problema, contrasta ideas en grupo, las defiende y reelabora con nuevas aportaciones, y toma decisiones, de modo similar a lo observado en el contexto universitario (Jiménez et al., 2013). En el caso de estas asignaturas de Educación Secundaria, puede plantearse de forma separada del aprendizaje de los contenidos, sirve para aplicar los conocimientos aprendidos y generar conocimientos significativos.

Hemos observado algunos aspectos de mucho interés a la hora del planteamiento de los casos: 1) Deben ser reales, es decir, la situación que se presenta debe ser posible, lógica y admisible. Si además se refiere a algún aspecto que ellos conozcan previamente, incrementa su interés e implicación, un aspecto que consideramos esencial en este nivel educativo base a los resultados obtenidos 2) Debe ser relevante para el alumnado, pues incrementa su implicación, 3) debe plantearse en un escenario problemático, es decir en una situación aún por resolver, 4) debe estar abierta a varias soluciones o hipótesis de trabajo, al objeto de fomentar la discusión en los grupos o entre ellos.

En ambas experiencias, hemos observado que el empleo de esta herramienta docente evita la adopción de una actitud pasiva y potencia la adquisición de las competencias propias de las materia en cuestión, además de potenciar la adquisición de otras competencias transversales como son la capacidad de análisis y síntesis, la capacidad de aplicar los conocimientos en la práctica, la resolución de problemas, la toma de decisiones o analizar, sintetizar y resolver problemas en cualquiera de los ámbitos empleados. En ambos casos, contrastamos que el alumno realmente aprende cuando asume una postura activa y esto sólo es posible cuando el profesor realiza una enseñanza activa. Para ello, es necesario que el alumno sea consciente de su papel en el aprendizaje (Martín y Ruiz, 2006).

Dentro del método de estudio de casos se pueden identificar cuatro fases. En la fase uno, de presentación, el profesor establece la escena, los objetivos de los estudios de caso y clarifica cualquier problema en el procedimiento. En esta fase, puede incluirse desde la presentación del contexto sanitario de los casos tratados en Enfermedades Infecciosas como la presentación de una situación económica concreta en la que se desarrolla el problema en cuestión. En la fase dos, de estudio individual, se les da a los estudiantes tiempo para leer, buscar información, plantear cuestiones, y anotar cualquier asunto que tenga relación. En la fase tres, de discusión, los estudiantes en grupos de tres a seis comparten puntos de vista, conocimientos y técnicas. Tienen que juzgar si la información dada es adecuada, luego desarrollar varias soluciones y elegir la mejor alternativa. En este punto, también es interesante reseñar que los alumnos deben "aprender" a utilizar las ventajas del acceso a la información que ofrece Internet, valorando la información que es útil y descartando la que no lo es. La fase cuarta es una sesión completa en la que cada grupo puede presentar su "mejor" solución y extraer, bajo la tutoría del profesor, los principios y problemas subyacentes. Una variante de este método es invitar a cada estudiante a preparar y presentar un estudio basado en su propia experiencia para discutir con el grupo o subgrupo. Como se observa, las posibilidades de aplicación son múltiples. Todos estos aspectos han sido revisados y comparados ampliamente en diversos trabajos (Fernández et al., 2006).

Según Fabra (1997), las ventajas del método son evidentes. En primer lugar, se aumenta la motivación del alumno por el tema de estudio, al confrontarle con situaciones reales, en consonancia con los datos que recogen diversos trabajos que inciden en la importancia del componente emocional (Fernández et al., 2006). Además, se potencian las habilidades del alumnado y se les acostumbra a trabajar en equipo. En efecto, gracias al fomento del 
diálogo y al aprendizaje mutuo entre estudiantes, el método permite desarrollar cualidades como técnicas de comunicación, de síntesis, de búsqueda e interpretación de la información, de negociación, de creatividad, de toma de decisiones etc. Además, el alumnado detecta la flexibilidad de los métodos de resolución y la existencia de más de una solución correcta al mismo problema. Los estudiantes perciben el aprendizaje como relevante y resaltan como puntos fuertes algunas de las características del APP. Utilizan más tiempo para el autoaprendizaje y hacen mayor uso de las TICs. Específicamente en el ámbito clínico, los estudiantes de APP son con frecuencia mejor considerados en sus conocimientos clínicos y habilidades, aunque sus conocimientos en ciencias básicas parezcan ser similares a los de los estudiantes en un curriculum tradicional (Albanese, 2000).

\section{Referencias}

Albanese, M. (2000). Problem-based learning: why curricula are likely to show little effect on knowledge and clinical skills. Med Educ, 34, 729-738

Alfonso-Roca, M.T. y Fonseca, M. (2001). Aprendizaje basado en problemas: situación actual y perspectivas de implantación en las Facultades de Medicina Europeas. Dolor, 16, 119-127.

Branda, L. A. (2004). El aprendizaje basado en problemas en la formación en Ciencias de la Salud. En: El aprendizaje basado en problemas: una herramienta para toda la vida. 2004. Agencia Laín Entralgo, Madrid.

De la Fe, C. y Corrales, J. C. (2007). Aplicación del método de estudio de casos en las prácticas de diagnóstico de la disciplina de Enfermedades Infecciosas. II Jornadas Nacionales sobre el EEES. Murcia.

Fabra, M. L. (1997). Notas del curso: el método del caso. Valencia: Instituto de Ciencias de la Educación de la U.P.V.

Fernández, M., García, J. N., Caso, A., Fidalgo, R. y Arias, O. (2006). El aprendizaje basado en problemas: revisión de estudios empíricos internacionales. Revista de Educación, 341, 397418.

García Sanz, M. P. (2012). Aprendizaje y evaluación de competencias. Formación por Centros. Murcia: Centro de Formación y desarrollo profesional. Universidad de Murcia.

Jiménez, J., Lagos, G. y Jareño, F. (2013). El aprendizaje basado en problemas como instrumento potenciador de las competencias transversales. Revista electrónica sobe la enseñanza de la economía pública, 13, 44-68.

Martín, C. y Ruiz, E. (2006). El aprendizaje de los estudiantes. Murcia: Plan de formación permanente de la Universidad de Murcia

Mateo, J. y Vlachopoulos, D. (2012). Aplicando la metodología del aprendizaje basado en problemas en la asignatura de Gestión Cultural: un modelo alternativo de evaluación. Revista Iberoamericana de Educación, 58(2).

Perrenoud, P. H. (2004). Diez nuevas competencias para enseñar. Barcelona: Graó. 


\section{Autores}

Christian de la Fe Rodríguez

Doctor en Veterinaria por la Universidad de Las Palmas de Gran Canaria, Profesor Titular de Sanidad Animal de la Facultad de Veterinaria de la Universidad de Murcia, Formador de Formadores del Centro de Formación de Profesorado de la Universidad de Murcia y Vicedecano de la Facultad desde el año 2012. Ha publicado más de 60 trabajos en revistas del JCR especialmente en el área de Veterinaria. Sus líneas de investigación se orientan al estudio de las micoplasmosis animales y otras enfermedades infecciosas de los rumiantes. A nivel docente, sus trabajos se orientan hacía la aplicación de metodologías activas de aprendizaje aplicables a la docencia de las Enfermedades Infecciosas (Sanidad Animal).

Ángel Gómez Martín

Doctor en Veterinaria por la Universidad de Murcia, Investigador Contratado de Sanidad Animal. Profesor asociado de la Facultad de Veterinaria durante 3 cursos académicos, ha publicado más de 20 trabajos en revistas del JCR especialmente en el área de Veterinaria. Sus líneas de investigación se orientan al estudio de las micoplasmosis animales y la epidemiología molecular.

\section{Juan Carlos Corrales Romero}

Doctor en Veterinaria por la Universidad de Murcia, Profesor Titular de Sanidad Animal de la Facultad de Veterinaria y Director del Departamento de Sanidad Animal desde 2013. Ha publicado más de 50 trabajos en revistas del JCR especialmente en el área de Veterinaria. Sus líneas de investigación se orientan al estudio de las micoplasmosis animales y otras enfermedades infecciosas de los rumiantes. A nivel docente, sus trabajos se orientan hacía la aplicación de metodologías activas de aprendizaje aplicables a la docencia en Medicina Preventiva y Policía Sanitaria (Sanidad Animal). 